TRANSACTIONS OF THE

AMERICAN MATHEMATICAL SOCIETY

Volume 362, Number 3, March 2010, Pages 1413-1421

S 0002-9947(09)04745-X

Article electronically published on September 18, 2009

\title{
THE SECOND CLOSED GEODESIC ON FINSLER SPHERES OF DIMENSION $n>2$
}

\author{
HANS-BERT RADEMACHER
}

\begin{abstract}
We show the existence of at least two geometrically distinct closed geodesics on an $n$-dimensional sphere with a bumpy and non-reversible Finsler metric for $n>2$.
\end{abstract}

\section{INTRODUCTION}

Estimates for the number of closed extremals for a one-dimensional variational problem on $n$-spheres have been studied extensively in the calculus of variations. From the geometric point of view one is looking for lower bounds for the number of geometrically distinct closed geodesics on $n$-dimensional spheres $S^{n}$ carrying a Finsler metric; cf. An75]. In [LF51 Lyusternik and Fet prove the existence of one closed geodesic for a Finsler metric on a compact and simply connected manifold based on ideas of Birkhoff. For further existence results a fundamental difference between Riemannian metrics, resp. reversible Finsler metrics, and non-reversible Finsler metrics occurs. On one hand there exist non-reversible Finsler metrics on $S^{2 n}$, resp. $S^{2 n-1}$, with only $2 n$ geometrically distinct closed geodesics. These metrics occur in the work of Katok, and their geometry is studied by Ziller in Zi82. On the other hand there is no example of a reversible Finsler metric, resp. a Riemannian metric, on $S^{n}$ with only finitely many closed geodesics. In fact on the two-sphere there are infinitely many geometrically distinct closed geodesics for every Riemannian metric due to the work of Bangert Ba93 and Franks Fr92, as well as Hingston Hi93.

In this paper we consider the following non-degeneracy assumption: We call a Finsler metric bumpy if all closed geodesics are non-degenerate, i.e., if there are no non-trivial periodic Jacobi fields orthogonal to the closed geodesic. For a bumpy metric the energy functional on the space of free loops is a Morse function with only non-degenerate critical $S^{1}$-orbits. This is a generic assumption, and the abovementioned Katok examples with only finitely many closed geodesics are bumpy.

The author showed in Ra89, ch. 4] that a bumpy Finsler metric on $S^{2}$ has at least two geometrically distinct closed geodesics. Instead of the non-degeneracy assumption one can prove the existence of two closed geodesics on $S^{2}$, resp. several geodesics on $S^{n}$, with length estimates also for an open set of Finsler metrics defined, for example, in terms of the flag curvature and the reversibility, cf. Ra07. In their recent work BL07] Bangert and Long prove that for every non-reversible Finsler metric on $S^{2}$ there are two closed geodesics. From results by Hofer, Wysocki,

Received by the editors August 9, 2006 and, in revised form, January 31, 2008

2000 Mathematics Subject Classification. Primary 53C22, 53C60, 58E10.

(C)2009 American Mathematical Society 
and Zehnder in HWZ03 one obtains the following statement: A bumpy metric on $S^{2}$ for which the stable and unstable manifolds of all closed geodesics intersect transversally carries either two or infinitely many geometrically distinct closed geodesics.

For the $n$-sphere of dimension $n \geq 3$ there are only results for generic metrics or metrics with curvature restrictions. Fet proves in Fe65 that every bumpy and reversible Finsler metric on a compact manifold carries at least two geometrically distinct closed geodesics. For results on the existence of infinitely many closed geodesics for generic metrics on spheres, resp. compact and simply connected manifolds, we refer to [Hi84, Ra94, and [Zi82, p. 141]. For further details and references we refer to the surveys [Ba85], Ta92, and [Lo06].

In this paper we prove for bumpy metrics the following extension of the result by Bangert and Long to all dimensions:

Theorem. Let a compact and simply connected manifold of the rational homotopy type of an $n$-dimensional sphere $S^{n}, n \geq 3$, carry a bumpy and non-reversible Finsler metric. Then there are at least two geometrically distinct closed geodesics.

The main ingredients of the proof are the relation between the average indices of closed geodesics for metrics with only finitely many closed geodesics shown in [Ra89, Thm. 3.1] and a detailed analysis of the sequence of Morse indices ind $\left(c^{m}\right)$ of the coverings $c^{m}$ of a prime closed geodesic $c$ using a formula due to Bott Bo56] as well as a careful discussion of the Morse inequalities. For $n \geq 4$ we use in addition the following indirect argument: If there is only one geometric closed geodesic represented by the prime closed geodesic $c$, then it is crucial that we are able to show in Proposition 2 that the sequence ind $\left(c^{m}\right), m \geq 1$, of Morse indices is monotone increasing. Using the Morse inequalities, we conclude that the difference ind $\left(c^{m+1}\right)-$ ind $\left(c^{m}\right) \leq 2$ for all $m \geq 1$. But since ind $(c)=n-1$, the common index jump theorem due to Long and Zhu (cf. [LZ02, Thm. 4.3]) implies that there are integers $k$ with ind $\left(c^{2 k+1}\right)-$ ind $\left(c^{2 k-1}\right)=2 \operatorname{ind}(c)=2 n-2 \geq 6$.

Note added in proof: The same result with a different proof was obtained independently in H. Duan and Y. Long: Multiple closed geodesics on bumpy Finsler spheres. J. Diff. Eq. 233 (2007), 221-240.

\section{Critical point theory}

Closed geodesics on a compact manifold with a Finsler metric $F$ can be characterized as the critical points of the energy functional

$$
E: \Lambda M \rightarrow \mathbb{R}, \quad E(\gamma)=\frac{1}{2} \int_{0}^{1} F^{2}\left(\gamma^{\prime}(t)\right) d t .
$$

Here $\Lambda M$ is the free loop space consisting of closed $H^{1}$-curves $\gamma: S^{1}:=[0,1] /\{0,1\}$ $\rightarrow M$ on the manifold $M$. For the case of a Riemannian manifold cf. Kl78 for the case of a Finsler metric cf. Ra92, ch. 5], Ra04, ch. 2]. References for facts from Finsler geometry are [BCS, CS05]. On $\Lambda M$ there is an $S^{1}$-action $(u, \gamma) \in S^{1} \times$ $\Lambda M \mapsto u \cdot \gamma \in \Lambda M, u . \gamma(t)=\gamma(t+u), t \in S^{1}$, leaving the energy functional invariant. In addition there is the mapping ${ }^{m}: \gamma \in \Lambda M \mapsto \gamma^{m} \in \Lambda M, \gamma^{m}(t)=\gamma(m t), t \in S^{1}$, and $E\left(\gamma^{m}\right)=m^{2} E(\gamma)$. Here $\gamma^{m}$ is the $m$-fold cover of $\gamma$. A closed geodesic $c$ is called prime if there is no closed geodesic $c_{1}$ and no integer $m>1$ with $c=c_{1}^{m}$.

We call a Finsler metric $F$ reversible if for all tangent vectors $X$ we have $F(-X)=F(X)$. Otherwise we call the metric non-reversible. We call two closed 
geodesics $c_{1}, c_{2}: S^{1} \rightarrow M$ of a non-reversible Finsler metric on a differentiable manifold $M$ geometrically equivalent if their traces $c_{1}\left(S^{1}\right)=c_{2}\left(S^{1}\right)$ coincide and if their orientations coincide. The equivalence class is also called a geometric closed geodesic. For a closed geodesic $c_{1}$ there is a prime closed geodesic $c$ such that the set of all geometrically equivalent closed geodesics consists of $u . c^{m}, m \geq 1, u \in S^{1}$. Let $\theta: \Lambda M \rightarrow \Lambda M$ be orientation reversing; i.e., $(\theta c)(t)=c(1-t)$. For a non-reversible Finsler metric this mapping in general does not leave the energy functional invariant, and in general for a closed geodesic $c$ the curve $\theta c$ is not a geodesic.

The second order behaviour of the energy functional in a neighborhood of a closed geodesic is determined by its index form $\mathcal{H}_{c}$ which equals the hessian $d^{2} E(c)$ of the energy functional by the second variational formula; cf. [Ra04, ch. 2]. The index ind $(c)$ of the closed geodesic $c$ is the index of the index form $\mathcal{H}_{c}$, i.e., it is the maximal dimension of a subspace on which $\mathcal{H}_{c}$ is negative definite. The nullity $\operatorname{null}(c)$ is the nullity of the index form $\mathcal{H}_{c}$ minus 1 . This convention is used since due to the $S^{1}$-action the nullity of the index form $\mathcal{H}_{c}$ is at least 1 . We call a Finsler metric bumpy if all closed geodesics are non-degenerate; i.e., for all closed geodesics $c$ the nullity $\operatorname{null}(c)=0$ vanishes.

For a closed geodesic $c$ let $\Lambda(c):=\{\gamma \in \Lambda M \mid E(\gamma)<E(c)\}$. We call

$$
\bar{C}_{*}(c):=H_{*}\left(\left(\Lambda(c) \cup S^{1} . c\right) / S^{1}, \Lambda(c) / S^{1} ; \mathbb{Q}\right)
$$

the $S^{1}$-critical group of $c$; cf. Ra92, ch. 6.3]. Here $H_{*}$ is the singular homology; as coefficient field we use in this paper the rationals $\mathbb{Q}$. Let $w_{k}(c):=\operatorname{dim} \bar{C}_{k}(c)$.

For the Morse theory of the energy functional it is important to describe the $S^{1}$ critical groups $\bar{C}_{*}\left(c^{m}\right)$ of the infinitely many critical points $S^{1} \cdot c^{m}, m \geq 1$, produced by a single prime closed geodesic. Therefore the sequence ind $\left(c^{m}\right), m \geq 1$, of the sequence of iterates $c^{m}$ is of great importance.

Definition 1 (Cf. Ra89, Def. 1.6]). For a closed geodesic $c$ let $\gamma_{c} \in\{ \pm 1 / 2, \pm 1\}$ be the invariant defined by $\gamma_{c}>0$ if and only if ind $(c)$ is even and $\left|\gamma_{c}\right|=1$ if and only if $\operatorname{ind}\left(c^{2}\right)-\operatorname{ind}(c)$ is even.

Then we obtain the following crucial

Lemma 1 (Cf. [Ra89, Prop. 2.2]). Let $c$ be a prime and non-degenerate closed geodesic, $m \geq 1$. Then for all $k \geq 0$,

$$
w_{k}\left(c^{m}\right)= \begin{cases}1, & k=\operatorname{ind}\left(c^{m}\right), m \text { even or } \gamma_{c}= \pm 1, \\ 0, & \text { otherwise }\end{cases}
$$

It follows from Bott's iteration formula [Bo56, Thms. A, C], resp. equation (9), that the sequence ind $\left(c^{m}\right), m \geq 1$, grows almost linearly. Therefore the average index

$$
\alpha_{c}:=\lim _{m \rightarrow \infty} \frac{\operatorname{ind}\left(c^{m}\right)}{m}
$$

is well defined and one can show that

$$
\left|\operatorname{ind}\left(c^{m}\right)-m \alpha_{c}\right| \leq n-1
$$

for all $m \geq 1$, where $n$ is the dimension of the manifold; cf. [Ra89, (1.4)].

Now we consider bumpy metrics with only a single geometric closed geodesic. Hence there is a prime closed geodesic $c$ such that any closed geodesic on $M$ is of 
the form $u \cdot c^{m}$ for some $u \in S^{1}$ and $m \geq 1$. Then we define for every $k \geq 0$,

$$
w_{k}:=\sum_{m=1}^{\infty} w_{k}\left(c^{m}\right)
$$

This number gives the number of critical orbits $S^{1} \cdot c^{m}$ whose $S^{1}$-critical group is non-trivial in dimension $k$. The Morse inequalities relate these local invariants of the critical points to the Betti numbers

$$
b_{k}:=b_{k}\left(\Lambda M / S^{1}, \Lambda^{0} M / S^{1} ; \mathbb{Q}\right)
$$

of the pair $\left(\Lambda M / S^{1} ; \Lambda^{0} M / S^{1}\right)$ of the quotient space $\Lambda M / S^{1}$ of the free loop space $\Lambda M$ divided by the $S^{1}$-action and the space $\Lambda M^{0}:=\{c \in \Lambda M ; E(c)=0\}$ of point curves. This space can be identified with the manifold $M$; it is the fixed point set of the $S^{1}$-action. In particular we will use the following Betti numbers of the quotient space $\Lambda S^{n} / S^{1}$ :

Proposition 1 (Cf. Ra89, Thm. 2.4]). If $M$ is a simply connected compact manifold rationally homotopy equivalent to the $n$-dimensional sphere $S^{n}$, then the Betti numbers $b_{k}:=b_{k}\left(\Lambda M / S^{1}, \Lambda^{0} M / S^{1} ; \mathbb{Q}\right)$ of the quotient $\Lambda M / S^{1}$ of the free loop space by the $S^{1}$-action satisfy:

(1) $b_{k} \in\{0,1,2\}$, and $b_{k} \geq 1$ if and only if $k \equiv n-1(\bmod 2)$ and $k \geq n-1$.

(2) If $n \equiv 0(\bmod 2)$, then $b_{k}=2$ if and only if $k=(2 j+1)(n-1), j \geq 1$.

(3) If $n \equiv 1(\bmod 2)$, then $b_{k}=2$ if and only if $k=j(n-1), j \geq 2$.

This proposition follows from the form of the Poincaré polynomials $P(t)=$ $\sum_{k} b_{k} t^{k}$ determined in [Ra89, (2.4),(2.5)]:

$$
P\left(\Lambda S^{n} / S^{1}, \Lambda^{0} S^{n} ; \mathbb{Q}\right)(t)=\left\{\begin{array}{lll}
t^{n-1}\left\{\frac{1}{1-t^{2}}+\frac{t^{2 n-2}}{1-t^{2 n-2}}\right\}, & n \equiv 0 \quad(\bmod 2), \\
t^{n-1}\left\{\frac{1}{1-t^{2}}+\frac{t^{n-1}}{1-t^{n-1}}\right\}, & n \equiv 1 \quad(\bmod 2) .
\end{array}\right.
$$

It follows that $B(n, 1):=\lim _{N \rightarrow \infty} \sum_{j=0}^{N}(-1)^{j} b_{j}$ satisfies

$$
B(n, 1)=\left\{\begin{array}{ccc}
-\frac{n}{2 n-2}, & n \equiv 0 \quad(\bmod 2) \\
\frac{n+1}{2 n-2}, & n \equiv 1 \quad(\bmod 2) .
\end{array}\right.
$$

Then we collect the following conclusions from the results in Ra89, (2.3),(2.6), Thm. 3.1(a)], resp. Ra92]:

Proposition 2. Let $F$ be a bumpy Finsler metric on a compact and simply connected manifold of the rational homotopy type of an $n$-sphere with only one geometric closed geodesic represented by the prime closed geodesic $c$.

(a) The average index $\alpha_{c}$ and the invariant $\gamma_{c}$ satisfy

$$
(-1)^{n-1} \frac{\alpha_{c}}{\gamma_{c}}=\left\{\begin{array}{ccc}
2-\frac{2}{n}, & n \equiv 0 & (\bmod 2), \\
2-\frac{4}{n+1}, & n \equiv 1 \quad(\bmod 2) .
\end{array}\right.
$$

(b) The numbers $w_{k}, k \geq 0$, of critical $S^{1}$-orbits whose $S^{1}$-critical group is non-trivial in dimension $k$ (cf. equation (2)) are bounded and there is a 
sequence $\left(q_{k}\right)_{k \geq 0}$ of non-negative integers satisfying

$$
w_{k}=b_{k}+q_{k}+q_{k-1}
$$

for all $k \geq 0$.

\section{BotT'S ITERATION FORMULA AND THE INDEX GROWTH}

For a closed geodesic $c: \mathbb{R} \rightarrow M$ with $c(t+1)=c(t)$ for all $t \in \mathbb{R}$ we define the linearized Poincaré mapping $P_{c}$ : For $p=c(0)$ let $V$ be the $(n-1)$-dimensional orthogonal complement in the tangent space $T_{p} M$ to the 1-dimensional subspace generated by $c^{\prime}(0)$ (with respect to the osculating Riemannian metric defined by the velocity field $c^{\prime}$ ). Then let

$$
P_{c}: V \oplus V \rightarrow V \oplus V, \quad P_{c}(X, Y)=\left(J(1), \frac{\nabla}{d t} J(1)\right)
$$

where $J$ is the Jacobi field determined uniquely by the initial conditions $J(0)=$ $X, \frac{\nabla}{d t} J(0)=Y$ along $c \mid[0,1]$. Here $\frac{\nabla}{d t}$ is the covariant derivative along $c$. The canonical symplectic structure on $V \oplus V$ is preserved by $P_{c}$; hence by choosing an orthonormal basis in $V$, we consider $P_{c}$ as an element of the group $\operatorname{Sp}(n-1)$ of linear symplectic maps of $\mathbb{R}^{n-1} \oplus \mathbb{R}^{n-1}$, which is well defined up to conjugation, i.e., independent of the choice of $p=c(0)$ and the choice of an orthonormal basis of $V$.

We denote by $\operatorname{Spec}(P) \subset \mathbb{C}$ the set of eigenvalues of the complexification $P$ of $P_{c}$ and denote by $V(z)=\operatorname{ker}(P-z I d)^{n-1}$ the generalized eigenspace for $z \in \operatorname{Spec}(P)$. Then

$$
N_{c}(z):=\operatorname{dim}_{\mathbb{C}}\left(P_{c}-z I d\right) \leq \operatorname{dim}_{\mathbb{C}} V(z)
$$

is called the $z$-nullity of $c$. Since $P$ is symplectic, with $z \in \operatorname{Spec}(P)$ also $z^{-1}, \bar{z}, \bar{z}^{-1} \in$ $\operatorname{Spec}(P)$. If $c, c^{2}$ are non-degenerate (i.e., $1,-1 \notin \operatorname{Spec}(P)$ ), it follows:

$$
\sum_{z \in \operatorname{Spec}(P),|z|=1, \operatorname{Im} z>0} N_{c}(z) \leq n-1 .
$$

In addition to the $z$-nullity $N_{c}(z)$ for a closed geodesic $c$ Bott defined the $z$-index $I_{c}:\{z \in \mathbb{C} ;|z|=1\} \rightarrow \mathbb{N}_{0}$. Both functions have the following properties (cf. Bo56, Thms. A, C], [Ra92, ch. 4]): The functions $I_{c}, N_{c}$ are invariant under conjugation; i.e., $I_{c}(\bar{z})=I_{c}(z), N_{c}(\bar{z})=N_{c}(z)$ for all $z$. The function $I_{c}$ is a continuous and constant function outside the set $\operatorname{Spec}\left(P_{c}\right)$. The finite jumps define the splitting numbers

$$
S_{c}^{ \pm}(z)=\lim _{\phi \rightarrow \pm 0} I_{c}(z \exp (i \phi))-I_{c}(z)
$$

satisfying

$$
0 \leq S_{c}^{ \pm}(z) \leq N_{c}(z) .
$$

The functions $I_{c}$ allow the following formula due to Bott [Bo56, Thms. A, C] for the sequence ind $\left(c^{m}\right)$ of the indices of the multiples $c^{m}$ of a closed geodesic $c$ :

$$
\operatorname{ind}\left(c^{m}\right)=\sum_{z^{m}=1} I_{c}(z)
$$

As an immediate consequence we obtain that for all $m \geq 1$,

$$
\operatorname{ind}\left(c^{m}\right) \geq \operatorname{ind}(c) \text {. }
$$


It was shown by Bott that the splitting numbers $S_{c}^{ \pm}(z)$ only depend on the conjugacy class of the linearized Poincaré mapping in the symplectic group.

If $c$ is a closed geodesic of a bumpy metric and $z_{1}=\exp \left(2 \pi i t_{1}\right), \ldots, z_{l}=$ $\exp \left(2 \pi i t_{l}\right)$ are the eigenvalues $z$ of the linearized Poincaré mapping with $|z|=1$, $\operatorname{Im}(z)>0$, and $0=t_{0}<t_{1}<t_{2}<\ldots<t_{l}<t_{l+1}=1 / 2$, then $l \leq n-1$ and the numbers $t_{j}$ are irrational since null $\left(c^{m}\right)=\sum_{z^{m}=1} N_{c}(z)$.

With the help of the function $I_{c}$ we get the following expression for the average index. Let $I_{1}=I_{c}(0)=I_{c}(\exp (2 \pi i t)), t \in\left[0, t_{1}\right)$, and for $j \in\{1,2, \ldots, l\}, I_{j}:=$ $I_{c}(\exp (2 \pi i t)), t \in\left(t_{j-1}, t_{j}\right)$, and $I_{l+1}:=I_{c}(-1)=I_{c}(\exp (2 \pi i t)), t \in\left(t_{l}, 1 / 2\right]$. Hence $\left\{I_{c}(\exp (z)) \mid z \in S^{1}-\operatorname{Spec}\left(P_{c}\right)\right\}=\left\{I_{1}, I_{2}, \ldots, I_{l+1}\right\}$. Then Bott's formula (9) implies

$$
\alpha_{c}=\int_{0}^{1} I_{c}(\exp (2 \pi i t)) d t=I_{1} t_{1}+\sum_{j=2}^{l} I_{j}\left(t_{j}-t_{j-1}\right)+I_{l+1}\left(\frac{1}{2}-t_{l}\right) .
$$

Since $I_{j}-I_{j+1}=S^{-}\left(z_{j}\right)-S^{+}\left(z_{j}\right)$, we obtain from equation (8) $\left|I_{j}-I_{j+1}\right| \leq N_{c}\left(z_{j}\right)$ where $N_{c}\left(z_{j}\right) \geq 1$. Then equation (7) implies

$$
\sum_{j=1}^{l}\left|I_{j}-I_{j+1}\right| \leq \sum_{j=1}^{l} N_{c}\left(z_{j}\right) \leq n-1 .
$$

The following proposition will be crucial in the proof of our theorem:

Proposition 3. Let $c$ be a closed geodesic of a bumpy Finsler metric on an $n$ dimensional manifold $M$. We denote by $z_{j}=\exp \left(2 \pi i t_{j}\right), t_{j} \in(0,1 / 2), j=1,2, \ldots, l$, $l \leq n-1$, the eigenvalues of the linearized Poincaré mapping $P_{c}$ whose imaginary part is positive with $0<t_{1}<t_{2}<\ldots<t_{l}<1 / 2$. Let $t_{0}=0, t_{l+1}=1 / 2$, and $I_{j}:=I_{c}(\exp (2 \pi i t)), t \in\left(t_{j-1}, t_{j}\right), j=1,2, \ldots, l+1$.

Let ind $(c)=n-1$, ind $\left(c^{2}\right) \geq n$, and $\alpha_{c}<2\left|\gamma_{c}\right|$.

Then the invariants of the closed geodesic $c$ satisfy:

(a) $\gamma_{c}=(-1)^{n-1} ; \alpha_{c}>1$; ind $\left(c^{2}\right)=n+1$.

(b) $I_{c}(1)=I_{1}=n-1>I_{2}>\cdots>I_{l}=1 ; I_{l+1}=I_{c}(-1)=2$.

(c) For all integers $m \geq 1$, ind $\left(c^{m+1}\right) \geq \operatorname{ind}\left(c^{m}\right)$.

Remark 1. Since the number $\bar{S}:=\sum_{j=1}^{l}\left|S^{+}\left(z_{j}\right)-S^{-}\left(z_{j}\right)\right|=\sum_{j=1}^{l}\left|I_{j+1}-I_{j}\right|=$ $n-1$ is maximal, it follows from the formula for the splitting numbers depending on the symplectic normal form given for example in [BTZ82, 2.13] that the closed geodesic is of elliptic type; i.e., the linearized Poincaré mapping has a symplectic normal form consisting only of 2-dimensional rotations.

Proof. We define

$$
I_{\min }:=\min \left\{I_{1}, I_{2}, \ldots, I_{l}\right\}, \quad r:=\min \left\{j \in\{1,2, \ldots, l\} \mid I_{j}=I_{\min }, 1 \leq j \leq l\right\} .
$$

Since ind $(c)=n-1$, we have $\gamma_{c}(-1)^{n-1} \in\{1 / 2,1\}$. If $\gamma_{c}=(-1)^{n-1} / 2$, then $\alpha_{c}<1$ by Proposition 2(a); hence $I_{\min }=0$ by equation (11). Then equation (12) implies that $r=l, I_{l+1}=0$. Bott's formula (9) then implies that ind $\left(c^{2}\right)=n-1+I_{l+1}=$ $n-1$, contradicting the assumption $\operatorname{ind}\left(c^{2}\right) \geq n$.

Hence we showed that $\gamma_{c}=(-1)^{n-1}$. By Bott's formula (9) we conclude that $\operatorname{ind}\left(c^{2}\right)=n-1+I_{l+1}$; hence by Definition 1 and the assumption $\operatorname{ind}\left(c^{2}\right) \geq n$, $I_{l+1}$ is an even and positive number. Since $\alpha_{c}<2$, equation (11) implies that 
$I_{\min }=I_{r} \leq 1$. From $I_{1}=n-1$ we conclude $I_{r} \in\{0,1\}$. If $I_{r}=0$, then equation (12) implies that $r=l+1$ and therefore $\operatorname{ind}\left(c^{2}\right)=n-1$, contradicting our assumption.

Hence we conclude $I_{r}=1$. Equation (12) implies that $r \in\{l, l+1\}$ and $I_{l+1}-I_{l} \leq$ 1 if $r=l$. Since $I_{l+1}$ is even and positive, we have $r=l$ and therefore $I_{l+1}=2$. Since $I_{1}-I_{l}=n-2$ and $I_{l+1}-I_{l}=1$ in equation (12), the inequalities are actually equalities, and hence $n-1=I_{1}>I_{2}>\cdots>I_{l}=1$ and $I_{l+1}=2$, which finishes the proof of parts (a) and (b).

It remains to prove part (c): We use the notation $e(x):=\exp (2 \pi i x)$. Using Bott's formula (9) and $I_{c}(-1)=2$, we obtain

$$
\operatorname{ind}\left(c^{m+1}\right)-\operatorname{ind}\left(c^{m}\right)=A_{m}+B_{m}
$$

with

$$
A_{m}:=\left\{\begin{array}{clc}
2, & m \equiv 1 \quad(\bmod 2), \\
2 I_{c}\left(e\left(\frac{m}{2 m+2}\right)\right)-2, & m \equiv 0 \quad(\bmod 2)
\end{array}\right.
$$

and

$$
B_{m}:=2 \sum_{1 \leq j<m / 2}\left\{I_{c}\left(e\left(\frac{j}{m+1}\right)\right)-I_{c}\left(e\left(\frac{j}{m}\right)\right)\right\} .
$$

From part (b) we conclude that $I_{c}(e(j /(m+1)))-I_{c}(e(j / m))<0$ for some $j \in \mathbb{N}$, $j<m / 2$, implies $j \in J_{m}:=\left\{p \in \mathbb{N} ; p /(m+1)<t_{l}<p / m, p<m / 2\right\}$ and therefore $I_{c}(e(j /(m+1)))-I_{c}(e(j / m))=-1$. On the other hand $J_{m}$ is either empty or consists of a single element $J_{m}=\left\{j_{m}\right\}$. Hence $B_{m} \geq-2$ and $B_{m}=-2$ if and only if $J_{m}=\left\{j_{m}\right\}$. Therefore we obtain immediately that $A_{m}+B_{m} \geq 0$ for odd $m$. Let $m$ be even. Since $I_{\min }=1$, we have $A_{m} \geq 0$. On the other hand $A_{m}=0$ implies that $m /(2 m+2)<t_{l}<1 / 2$. Hence $J_{m}=\emptyset$ and $A_{m}+B_{m} \geq 0$. If $A_{m}>0$, then $A_{m} \geq 2$; hence also in this case $A_{m}+B_{m} \geq 0$. Therefore we obtain in all cases ind $\left(c^{m+1}\right)-\operatorname{ind}\left(c^{m}\right)=A_{m}+B_{m} \geq 0$.

We use the common index jump theorem due to Y. Long and C. Zhu for a single closed geodesic $c$ of a bumpy metric. For a closed geodesic $c$ of a bumpy metric the nullities null $\left(c^{m}\right)$ for all $m$ vanish as well as the splitting number $S_{c}^{+}(1)$. Hence we obtain as a particular case of the common index jump theorem the following proposition. Here we note that the common index jump theorem also applies to the closed geodesic problem on Finsler manifolds; cf. [Lo02, Rem. 12.2.5].

Proposition 4 ([LZ02, Thm. 4.3] [Lo2, Thm. 11.2.1]). Let c be a closed geodesic of a bumpy Finsler metric with positive average index $\alpha_{c}$. Then there are infinitely many $k$ with

$$
\text { ind }\left(c^{2 k+1}\right)-\operatorname{ind}\left(c^{2 k-1}\right)=2 \operatorname{ind}(c) \text {. }
$$

\section{Proof of the theorem}

We assume that there is a prime closed geodesic $c$ such that all closed geodesics on $M$ are geometrically equivalent to $c$ and $n=\operatorname{dim} M \geq 3$. For the average index $\alpha_{c}$ and the invariant $\gamma_{c}$ we obtain from Proposition $2(\mathrm{a})$ :

$$
1 \leq \frac{\alpha_{c}}{\left|\gamma_{c}\right|}<2, \quad \alpha_{c}=\left|\gamma_{c}\right| \Leftrightarrow n=3
$$


Let $w_{k}:=\sum_{m=1}^{\infty} w_{k}\left(c^{m}\right)$; cf. equation (2). Since the Betti numbers $b_{k}=$ $b_{k}\left(\Lambda M / S^{1}, \Lambda^{0} M / S^{1} ; \mathbb{Q}\right)$ vanish in dimensions $k \equiv n(\bmod 2)$ (cf. Proposition 1), we conclude from the Morse inequalities (see (5) ) that the sequence $\left(q_{j}\right)_{j \geq 0}$ vanishes identically, i.e.,

$$
w_{k}=\#\left\{m \in \mathbb{N} ; \operatorname{ind}\left(c^{m}\right)=k, m \text { odd or } \gamma_{c}= \pm 1\right\}=b_{k} \in\{0,1,2\}
$$

for all $k \in \mathbb{N}_{0}$ with $b_{k} \in\{1,2\}$ if and only if $k \equiv n-1(\bmod 2)$ and $k \geq n-1$; cf. Proposition 1 and Lemma 1

We conclude from equation (10) and from $b_{0}=b_{1}=\ldots=b_{n-2}=0, b_{n-1}=1$ (cf. Proposition 11) that

$$
\operatorname{ind}(c)=n-1 .
$$

We conclude from equation (11) and equation (11) that $n-1=\operatorname{ind}(c) \leq \operatorname{ind}\left(c^{2}\right)<$ $2 \cdot 2+n-1=n+3$. If $\operatorname{ind}\left(c^{2}\right)=\operatorname{ind}(c)=n-1$, then Definition 1 and Lemma 1 imply $w_{n-1} \geq w_{n-1}(c)+w_{n-1}\left(c^{2}\right) \geq 2$, contradicting equation (17). Therefore $\operatorname{ind}\left(c^{2}\right) \geq n$, and we conclude from Proposition 3(a) that $\gamma_{c}=(-1)^{n-1}$ and $\alpha_{c}>1$. In dimension $n=3$ this yields a contradiction to equation (16) and finishes the proof.

Hence we can now assume $n \geq 4$ : Since the sequence ind $\left(c^{m}\right), m \geq 1$, is monotone increasing by Proposition 3 (c) and $b_{k}=w_{k} \geq 1$ for all $k \equiv n-1(\bmod 2), k \geq n-1$, we have $\operatorname{ind}\left(c^{m+1}\right)-\operatorname{ind}\left(c^{m}\right) \in\{0,2\}$ for all $m \geq 1$. Therefore for $n \geq 4$ we obtain for all $m \geq 1$,

$$
\operatorname{ind}\left(c^{m+2}\right)-\operatorname{ind}\left(c^{m}\right) \leq 4 .
$$

But we conclude from Proposition 4 that for $n \geq 4$ there is a $k_{1} \in \mathbb{N}$ with

$$
\operatorname{ind}\left(c^{2 k_{1}+1}\right)-\operatorname{ind}\left(c^{2 k_{1}-1}\right)=2 n-2 \geq 6,
$$

contradicting equation (19). Therefore we obtain a contradiction to our assumption made at the beginning of our proof.

Remark 2. It is likely that two is not the optimal lower bound for the number of closed geodesics of a non-reversible bumpy metric on $S^{n}, n \geq 3$. Katok's examples carry $2 n$ closed geodesics on $S^{2 n}$ and $S^{2 n-1}$. One can show that any non-reversible and bumpy Finsler metric sufficiently $C^{2}$-close to the standard Riemannian metric of constant curvature 1 on $S^{2 n}$, resp. $S^{2 n-1}$, carries at least $2 n$ closed geodesics of length approximately $2 \pi$; cf. [Zi82, p. 141]. Estimates on the number of closed geodesics under pinching assumptions for the flag curvature depending on the reversibility are presented for example in [Ra07, Thm. 3, Thm. 8]. Various questions, resp. conjectures, about the optimal lower bound for an arbitrary Finsler metric on $S^{n}$ are given in [Zi82, p. 155] and [Lo06, ch. 4].

\section{REFERENCES}

[An75] D.V. Anosov: Geodesics in Finsler geometry. (Russian) Proc. Intern. Congr. Mathem. (Vancouver, B.C., 1974), Vol. 2, Canad. Math. Congress, Montreal, Que. (1975), 293297= Amer. Math. Soc. Transl. 109 (1977), 81-85 MR0426058 (54:14004)

[BTZ82] W. Ballmann, G. Thorbergsson, and W. Ziller: Closed geodesics on positively curved manifolds. Ann. Math. (2) 116 (1982), 213-247 MR672836 (83k:58022)

[Ba85] V. Bangert: Geodätische Linien auf Riemannschen Mannigfaltigkeiten. Jber. d. Dt. Math.-Verein. 87 (1985), 39-66 MR789708|(87i:58036)

[Ba93] : On the existence of closed geodesics on two-spheres. Intern. J. Math. 4 (1993), 1-10 MR:1209957 (94d:58036) 
[BL07] V. Bangert and Y. Long: The existence of two closed geodesics on every Finsler 2sphere. arXiv:0709.1243

[BCS] D. Bao, S.S. Chern, and Z. Shen: An Introduction to Riemann-Finsler Geometry. Grad. Texts Mathem. 200, Springer Verlag, New York, 2000 MR:1747675 (2001g:53130)

[Bo56] R. Bott: On the iteration of closed geodesics and the Sturm intersection theory. Comm. Pure Appl. Math. 9 (1956), 171-206 MR0090730(19:859f)

[CS05] S. S.Chern and Z. Shen: Riemann-Finsler Geometry. Nankai Tracts Math. 6, World Scientific, Singapore, 2005 MR2169595 (2006d:53094)

[Fe65] A.I. Fet: A periodic problem in the calculus of variations. Dokl. Akad. Nauk SSSR (N.S.) 160 (1965), 287-289 (Russian) = Sov. Mathem. 6 (1965), 85-88. MR0220316 $(36: 3381)$

[Fr92] J. Franks: Geodesics on $S^{2}$ and periodic points of annulus homeomorphisms. Invent. Math. 108 (1992), 403-418 MR.1161099 (93f:58192)

[Hi84] N. Hingston: Equivariant Morse theory and closed geodesics. J.Differential Geom. 19 (1984), 85-116 MR739783(85i:58036)

[Hi93] _ On the growth of the number of closed geodesics on the two-sphere. Intern. Math. Res. Notices (1993), no. 9, 253-262 MR1240637 (94m:58044)

[HWZ03] H. Hofer, K. Wysocki, and E. Zehnder: Finite energy foliations of tight threespheres and Hamiltonian dynamics. Ann. Math. (2) 157 (2003), 125-255 MR1954266 (2004a:53108)

[Kl78] W. Klingenberg: Lectures on closed geodesics. Grundlehren der Math. Wiss. 230, Springer-Verlag, Berlin, Heidelberg, New York, 1978 MR 0478069 (57:17563)

[Lo02] Y. Long: Index Theory for Symplectic Paths with Applications. Progress Math. 207, Birkhäuser, Basel-Boston-Berlin, 2002 MR.1898560 (2003d:37091)

[Lo06] _ : Multiplicity and stability of closed geodesics on Finsler 2-spheres. J. Eur. Math. Soc. 8 (2006), 341-353 MR.2239281 (2007c:58021)

[LZ02] Y. Long and C. Zhu: Closed characteristics on compact convex hypersurfaces in $\mathbb{R}^{2 n}$. Ann. Math.(2) 155 (2002), 317-368 MR1906590(2003e:37083)

[LF51] L. Lyusternik and A.I. Fet: Variational problems on closed manifolds. Dokl. Akad. Nauk SSSR (N.S.) 81 (1951), 17-18. (Russian) MR0044760 (13:474c)

[Ra89] H.B. Rademacher: On the average indices of closed geodesics. J. Differential Geom. 29 (1989), 65-83 MR.978076 (90c:58038)

[Ra92] _ : Morse-Theorie und Geschlossene Geodätische. Bonner Math. Schriften 229 (1992), MR1237191 (94h:58049)

[Ra94] : On a generic property of geodesic flows, Math. Ann. 298 (1994), 101-116 MR.1252820 (94j:58040)

[Ra04] : A Sphere theorem for non-reversible Finsler metrics, Math. Ann. 328 (2004), 373-387 MR2036326 (2004j:53098)

[Ra07] _ Existence of closed geodesics on positively curved Finsler manifolds. Ergod. Th. \& Dyn. Syst. 27 (2007), 957-969 MR2322187 (2008d:53050)

[Ta92] I.A. Taimanov: Closed extremals on two-dimensional manifolds. Uspekhi Mat. Nauk 47 (1992), 143-185. (Russian) = Russ. Math. Surveys 47 (1992), 163-211 MR.1185286 (93k:58050)

[Zi82] W. Ziller: Geometry of the Katok examples. Ergod. Th. \& Dyn. Syst. 3 (1982), 135-157 MR743032 (86g:58036)

Mathematisches Institut, Universität Leipzig, 04081 Leipzig, Germany

E-mail address: rademacher@math.uni-leipzig.de 